\title{
Game theory in theology
}

\author{
H.N.V. Temperley
}

Superior Beings: If They Exist, How Would We Know? By Steven J. Brams.

Springer-Verlag: 1983. Pp.202. Pbk DM 32, \$12.50.

MANY scientists hold that no problem is so obscure, or so sacred, that it can be exempt from fearless rational enquiry. Game theory, created by such profound thinkers as von Neumann and Turing in the late 1940 s, attempts to codify, and to quantify where appropriate, the many ways in which two or more people or organisms can interact with one another. In this book, Steven Brams has applied its tenets to exploring the ramifications of the possible existence of "superior beings"; while I am not shocked by the title of his book, I am surprised that nothing of this kind seems to have been attempted before.

Many writers, myself included, have tried to look at the problems that would confront a "superior being" (Brams's expression) who was directing an organization as big as the Earth. It may well be intrinsically impossible for the superior being to allow for some free-will on the part of the inhabitants and at the same time ensure a fair distribution of rewards and punishments. And it may well be that the world is learning by its mistakes. Along such lines one can look for a partial solution of the problem of Evil.

The author confines himself almost entirely to the simplest possible type of "game" between an ordinary person $(\mathrm{P})$ and a superior being (SB), in which, at each play, each participant has only two possible courses of action available. Even under these severe restrictions, which obviously preclude consideration of some very important issues such as redemption, priesthood and the possible existence of the Devil, Brams is able to set up and consider a wide variety of intriguing questions, such as how could SB best "reveal"' himself to P and what policy $\mathrm{He}$ should follow if $\mathrm{P}$ commits "sin".

The principal difficulty with this approach is that scientists genuinely do not know what theologians actually mean by concepts such as omniscience and omnipotence. The author examines the effect of using various alternative definitions of his own, always taking great care to ensure that they are consistent with the exercise of free-will by $P$. Not the least interesting part of the investigation is the study of how changes in the definitions can affect the outcome of some of the games.

The newcomer to game theory will find many surprises in this book. Two participants can play no less than 78 different games under the severe restrictions mentioned above. As many as 57 of these can be described as mathematically nontrivial. In certain games, SB may be obliged to act capriciously (in game-theory language this is to use a mixed strategy) in order to obtain the best possible outcome. Even more surprising is the conclusion that in certain games the possession by SB of "superior" qualities can lead to a worse outcome for SB, if it is assumed that $\mathrm{P}$ knows about, or guesses at, those qualities.

We can discuss the game of "Chicken" as an illustration. This models two players who are on a collision course; the player who first gives way is considered to have lost face. Each player can choose between the two strategies of co-operation $(C)$ and non-cooperation $(N)$. The combined outcome $N N$ is regarded as a disaster by both players who both give it the rating 1 (worst). The outcome $C N$ is rated 2 (next worst) by the first player who chose $C$ but 4 (best) by the second player who chose $N$. The ratings of $N C$ are mirror images of those of $C N$, while the outcome $C C$ is rated 3 (second best) by both players. (A game would be trivial, "not worth playing", if both players gave the same rating to all four outcomes.)

This apparently straightforward game gives rise to a paradox. Brams gives a simple argument to show that the likely outcome of play between equals is $C C$. Now suppose that it is played between SB, who is omniscient in the sense that he can predict P's choice, and that $\mathbf{P}$ is aware of this. Now P will undoubtedly choose the strategy $N$. Why? Because SB, knowing this, will be obliged to choose $C$ to avoid the disastrous outcome $N N$.

Thus, in this not unrealistic model of a real encounter, SB's "omniscience" has

\section{Genetics and ecology}

\section{Nick Barton}

\section{The Evolutionary Biology of Colonizing} Species.

By P.A. Parsons.

Cambridge University Press: 1983.

Pp.262. £15, \$29.95.

How do plants and animals colonize new places, and new habitats? What are the consequences of colonization for the genetic structure of populations, and the ecological structure of communities? These are two questions which touch upon many difficult issues in evolutionary biology. For example, the boundary of a species will often be set by its ability to adapt to extreme environments; given the both damaged his chances and improved those of $\mathrm{P}$, the outcome $C N$ being rated $(2,4)$ in place of the $(3,3)$ obtained if played between equals! This is just one of many fascinating situations analysed by Brams. One can go on to consider what happens if a game is repeated and players are allowed to change their strategies. The conclusion must be that many of the difficulties facing an administrator of the world are encapsulated in a two-person game such as this.

Brams repeatedly refers to an earlier work, Biblical Games: A Strategic Analysis of Stories in the Old Testament (MIT Press, 1980), and reports his conclusion that the Jehovah of the Old Testament was "a superlative strategist... driven by very humanlike calculations ..."'. This earlier study has, apparently, greatly helped Brams in formulating the problems studied in the present book.

A few decades ago a work such as this would have been rejected (probably unread) as frivolous and profane. Had it appeared a little earlier still, the consequences might have been even more drastic. Brams is well aware of this and his introduction, explaining what he is trying to do, is a joy to read. His arguments, some of them quite complicated, are presented clearly and enough background information is given to enable the non-expert in game theory to follow what is going on.

The claim that the book makes a contribution to solving the problem of Evil is justified, but I can hold out no hope that certain aspects of it will ever be dealt with along such lines. Why, for example, did the horrors perpetrated by the Nazis not provoke a drastic divine intervention? And why have the people of Vietnam been punished for such a long time? Game theory cannot begin to answer such formidable questions as these.

H.N.V. Temperley was formerly Professor of Applied Mathematics at University College, Swansea.

rapid and extensive changes which can be effected by artificial selection, the definite borders of many species have seemed surprising, and have led to the idea that although peripheral populations could, if left to themselves, adapt and spread into unoccupied territory, the flow of genes from more central populations prevents adaptations to peripheral conditions, and so limits the range of the species. This now seems unlikely; there are many observations of differential adaptation in adjacent populations - for example, the sharp gradients in heavy-metal tolerance in grasses living on mines.

We are left, then, with the problem of understanding what restricts the power of natural selection in individual populations. In some sense, the limit must be set by lack of suitable genetic variation; but to what extent does the primary restriction come 